\title{
The vertical conductivity of aquifers
}

\author{
Yingwang Zhao ${ }^{1,2, a}$ \\ ${ }^{1}$ China University of Mining \& Technology, Beijing, Beijing 100083, China; \\ ${ }^{2}$ Guizhou Coal Mine Design \& Research Institute, Guiyang 550025, China. \\ ayingwangzhao@163.com
}

Keywords: vertical conductivity, numerical models, groundwater.

\begin{abstract}
The hydraulic parameters are important for the numerical computation in which hydraulic conductivity is one of the most frequent. The horizontal component of hydraulic conductivity could be obtained with field trial. And the vertical component is usually computed with the horizontal one by dividing a constant. However, this procedure always generates significant errors which are illustrated by the theoretical analysis and numerical comparison study. For practical engineering application, a brief algorithm to compute the vertical conductivity is proposed and checked by a numerical model.
\end{abstract}

\section{Introduction}

Hydraulic conductivity, symbolically represented as $K$, is a property of soils and rocks that describes the ease with which a fluid (usually water) can move through pore spaces or fractures. It depends on the intrinsic permeability of the material, the degree of saturation, and on the density and viscosity of the fluid [1]. The hydraulic conductivity is often considered as a vector in 3-D space and the horizontal component and the vertical one are different.

In practical groundwater engineering, the hydraulic conductivity is one of the most important parameters concerned to the computational accuracy. The horizontal conductivity could be obtained with field trial while the vertical one could not. And the vertical one is used to be computed with the horizontal one by dividing a constant, 2-10. However, the vertical one is usually much less than one tenth of the horizontal one when two or more materials are defined as one aquifer. Then, the vertical conductivities of those aquifers become larger and the hydraulic conductivities of aquitard, including horizontal component and vertical one, becomes less. In the other situations, the vertical conductivity of one model is assigned larger which make the fluxes through aquitard much larger. To solve this problem, the models with different materials are researched by theoretical and numerical computation.

\section{The parameters for different materials}

The hydraulic conductivity of aquifers is a complex physical quantity. It varies with the materials, the fracture development, the geologic structure and a lot of factors. In this study, we consider mainly the materials. And for different materials, we used the empirical value by Zheng [2] as shown in Table 1.

\begin{tabular}{ccc}
\multicolumn{3}{c}{ Table 1 Hydraulic conductivity of different materials (m/day) } \\
\hline Materials & Scope & Selected value \\
\hline Gravel & $25.9-2592$ & 250 \\
Grit & $7.8 \times 10^{-2}-518.4$ & 10 \\
Medium Sand & $7.8 \times 10^{-2}-43.2$ & 2 \\
Fine Sand & $1.7 \times 10^{-2}-17.3$ & 0.7 \\
Silt & $8.6 \times 10^{-5}-1.7$ & $1.5 \times 10^{-3}$ \\
Moraine & $8.6 \times 10^{-8}-0.2$ & $1.5 \times 10^{-4}$ \\
Clay & $8.6 \times 10^{-7}-4.3 \times 10^{-4}$ & $2 \times 10^{-5}$
\end{tabular}

The scope data is recomposed form Zheng and Bennett (2002). The selected value with the middle exponent will 
be used in the following computation.

For different materials, the ratios of vertical and horizontal conductivities are also different. Fetter [3] and Weeks [4] have researched the ratio with gravels and sands which are between 2 and 20. Kong [5] and many other engineers [6] discussed that ratio with clay. Its vertical conductivity is almost one fourth or tenth of that horizontal one. With the loess, the ratio is obvious different. The vertical one is bigger that are twice or triple horizontal conductivity [7-9]. And in the plain area, many researches have checked that it is reasonable for the ratio, one tenth, with single material [10-12]. In this study, we apply one fifth as the ratio for each single material.

\section{Theoretical analysis}

In theory, the vertical conductivity could be computed considering each material parameter with the following equation that

$$
K_{v}=\frac{\sum_{i=1}^{n} l_{i}}{\sum_{i=1}^{n} \frac{l_{i}}{K_{v i}}}
$$

where $K_{v}$ is the vertical conductivity of the whole aquifer and $K_{v i}$ is the vertical conductivity of material $i, l_{i}$ is the thickness of material $i$ and $n$ is the number of materials.

With the drill column as shown in Table 2, two aquifers and one aquitard are constructed. With the empirical conductivity and the ratio of vertical and horizontal conductivities of each material, the vertical conductivity of each aquifer and the aquitard could be computed according to equation (1). The corresponding data are shown in Table 3. In Table 3, the huge errors of vertical conductivity computed by conventional procedure are illustrated.

Table 2 The drill column and conductivity of each material

\begin{tabular}{|c|c|c|c|c|c|}
\hline Period & Number & Material & $\begin{array}{c}K_{m h} \\
\text { (m/day) }\end{array}$ & $\begin{array}{c}K_{m v} \\
\text { (m/day) }\end{array}$ & $\begin{array}{l}\text { Thickness } \\
\text { (m) }\end{array}$ \\
\hline Quaternary & 1 & Silt & $1.5 \times 10^{-3}$ & 0.0003 & 15 \\
\hline \multirow{2}{*}{ Neogene } & 2 & Fine Sand & 0.7 & 0.14 & 55 \\
\hline & 3 & Medium Sand & 2 & 0.4 & 10 \\
\hline \multirow{8}{*}{ Cretaceous } & 4 & Grit & 10 & 2 & 10 \\
\hline & 5 & Medium Sand & 2 & 0.4 & 10 \\
\hline & 6 & Silt & $1.5 \times 10^{-3}$ & 0.0003 & 50 \\
\hline & 7 & Fine Sand & 0.7 & 0.14 & 45 \\
\hline & 8 & Clay & $2 \times 10^{-5}$ & 0.000004 & 10 \\
\hline & 9 & Silt & $1.5 \times 10^{-3}$ & 0.0003 & 15 \\
\hline & 10 & Clay & $2 \times 10^{-5}$ & 0.000004 & 25 \\
\hline & 11 & Silt & $1.5 \times 10^{-3}$ & 0.0003 & 10 \\
\hline \multirow{5}{*}{ Jurassic } & 12 & Grit & 10 & 2 & 25 \\
\hline & 13 & Fine Sand & 0.7 & 0.14 & 10 \\
\hline & 14 & Gravel & 250 & 50 & 45 \\
\hline & 15 & Grit & 10 & 2 & 15 \\
\hline & 16 & Medium Sand & 2 & 0.4 & 30 \\
\hline
\end{tabular}

$K_{m h}$ is the horizontal conductivity of one material and $K_{m v}$ is the vertical conductivity of one material by dividing a constant, 5 , from the horizontal one. 
Table 3 The conceptualizing aquifers

\begin{tabular}{|c|c|c|c|c|c|}
\hline Number & Material & Aquifer Id & $\begin{array}{c}K_{a} \\
\text { (m/day) }\end{array}$ & $\begin{array}{c}K_{b} \\
\text { (m/day) }\end{array}$ & $\begin{array}{c}K_{c} \\
\text { (m/day) }\end{array}$ \\
\hline 1 & Silt & \multirow{7}{*}{ I } & \multirow{7}{*}{$9.0 \times 10^{-4}$} & \multirow{7}{*}{0.22} & \multirow{7}{*}{$2.2 \times 10^{-2}$} \\
\hline 2 & Fine Sand & & & & \\
\hline 3 & Medium Sand & & & & \\
\hline 4 & Grit & & & & \\
\hline 5 & Medium Sand & & & & \\
\hline 6 & Silt & & & & \\
\hline 7 & Fine Sand & & & & \\
\hline 8 & Clay & \multirow{4}{*}{ II } & \multirow{4}{*}{$6.8 \times 10^{-6}$} & \multirow{4}{*}{$1.30 \times 10^{-6}$} & \multirow{4}{*}{$1.30 \times 10^{-5}$} \\
\hline 9 & Silt & & & & \\
\hline 10 & Clay & & & & \\
\hline 11 & Silt & & & & \\
\hline 12 & Grit & \multirow{5}{*}{ III } & \multirow{5}{*}{0.75} & \multirow{5}{*}{18.75} & \multirow{5}{*}{1.88} \\
\hline 13 & Fine Sand & & & & \\
\hline 14 & Gravel & & & & \\
\hline 15 & Grit & & & & \\
\hline 16 & Medium Sand & & & & \\
\hline
\end{tabular}

$K_{a}$ is the vertical conductivity of one aquifer computed by equation (1); $K_{b}$ is the horizontal conductivity and $K_{c}$ is the vertical one computed by dividing a constant, 10 , from the horizontal one.

With equation (1) and Table 3, we could draw a conclusion that the vertical conductivity of an aquifer is mainly determined by the material with minimum vertical conductivity. Considering that the conductivities of different materials varies with exponent, when the thickness of the material with minimum vertical conductivity is not much less, the vertical conductivity of an aquifer could be computed briefly with that single material as

$$
K_{v}=\frac{\sum_{i=1}^{n} l_{i}}{\sum_{i=1}^{n} \frac{l_{i}}{K_{v i}}} \approx \frac{\sum_{i=1}^{n} l_{i}}{\sum_{j=1}^{m} l_{j}} K_{v i}
$$

where $m$ is the number of materials whose vertical conductivity is $K_{v i}$.

\section{Numerical models}

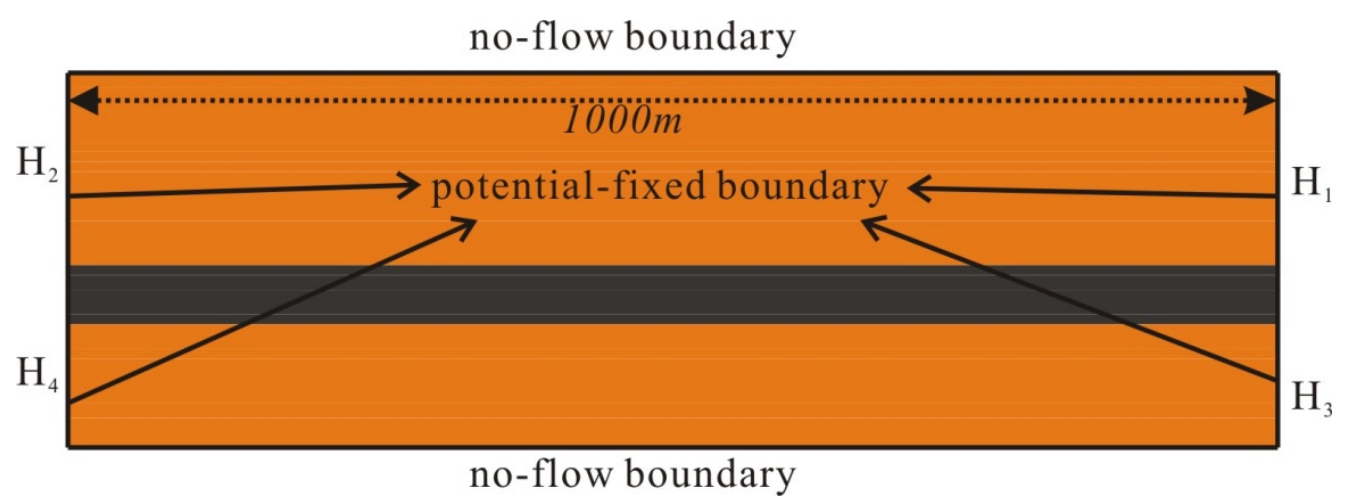

Figure 1 Model setting in lateral view.

The concept of the above two aquifer and one aquitard is expanded to a numerical model. Then the fluxes across the aquitard are computed with different vertical conductivities. Assumed that the leak fluxes form the upper top slice and the lower bottom slice are zero. The boundaries parallel to the profile in Figure 1 are both no-flow ones whose distance is $1000 \mathrm{~m}$. And the boundaries of those 
two aquifers are potential-fixed ones as shown in Figure 1. With different potential value for those potential-fixed boundaries, the flux is computed with MODFLOW. The fluxes and the potential assignment data are listed in Table 4. This comparison illustrates the huge error caused by the conventional procedure to compute vertical conductivity and the effectiveness of the brief algorithm, equation (2).

Table 4 The fluxes across the aquitard and the corresponding error

\begin{tabular}{ccccccc}
\hline $\begin{array}{l}\text { Methods to } \\
\text { compute } \boldsymbol{K}_{\boldsymbol{v}}\end{array}$ & $\boldsymbol{H}_{\mathbf{1}}(\mathbf{m})$ & $\boldsymbol{H}_{\mathbf{2}}(\mathbf{m})$ & $\boldsymbol{H}_{\mathbf{3}}(\mathbf{m})$ & $\boldsymbol{H}_{\mathbf{4}}(\mathbf{m})$ & Flux $\left(\mathbf{m}^{\mathbf{3}} / \mathbf{d a y}\right)$ & Error \\
\hline $\mathbf{1}$ & 400 & 390 & 390 & 380 & 1.1193 & $9.38 \%$ \\
$\mathbf{2}$ & 400 & 390 & 390 & 380 & 1.1291 & $9.14 \%$ \\
$\mathbf{3}$ & 400 & 390 & 390 & 380 & 2.1638 & $75.2 \%$ \\
\hline
\end{tabular}

The No. 1 method to compute $K_{v}$ follows equation (1); the No. 2 method follows equation (2) and the No. 3 method to compute $K_{v}$ means the conventional process by dividing a constant, 10 . The exact flux, $1.2351 \mathrm{~m}^{3} /$ day, is obtained by simulate the model with 16 layers that one material one layer. And the error means the relative error.

\section{Conclusion}

The conventional procedure to compute vertical conductivity form horizontal one by dividing a constant is not scientific and could generate huge errors. Only when the aquifer includes one single material, it could be valid. For general aquifers with different materials, the vertical conductivity could be computed briefly by the material with minimum vertical conductivity as shown in equation (2).

\section{Reference}

[1] Information on https://en.wikipedia.org/wiki/Hydraulic_conductivity.

[2] Zheng, C. M., and G. D. Bennett, Applied Contaminant Transport Modeling, second ed., Wiley, 2002.

[3] Fetter, C. W., Applied Hydrogeology, fourth ed., Prentice Hall, New Jersey, 2001.

[4] Weeks, A. G., The stability of natural slopes in the south-east England as affected by periglacial activity, Quarterly Journal of Engineering Geology and Hydrogeology, 1969, 1: 49-61.

[5] Kong. D. F., Geotechnical Engineering and Environmental Geology, Southwest Jiaotong University Press, Chengdu, 1999.

[6] Hebei Provincial Department of Land and Resources, and Hebei Oceanic Administration, The Oceanic Resource Survey and Evaluation of Hebei Province, Maritime Press, Beijing, 2007.

[7] Zhang Z. H., The Collapsibility and Permeability Characteristics of Loess in China, Geology of China, 1962, 12: 1-8.

[8] Wang S., and Tolo L., Geology and Geomorphology Tutorial, China Agricultural University Press, Beijing, 2005.

[9] Li P. C., The Conversion Mechanism and Regulation of Water Status in the Loess Plain Irrigation Zone, Shaanxi Science and Technology Press, Xi’an, 1999.

[10] Zhou X. Y., Li W. P., Groundwater Monitoring Information System Model and Sustainable Development, Science Press, Beijing, 2011.

[11] Li G. M., The Method and Application of Wellhead Protection Area Delineation, China Environmental Science Press, Beijing, 2011.

[12] Liu J. R., Proceedings of 2002 Beijing International Geothermal Symposium, Geological Publishing House, Beijing, 2002. 